\title{
Eosinophils contribute to innate antiviral immunity and promote clearance of respiratory syncytial virus
}

\author{
Simon Phipps, ${ }^{1-3}$ Chuan En Lam, ${ }^{1-3}$ Suresh Mahalingam, ${ }^{4}$ Matthew Newhouse, ${ }^{1,2}$ Ruben Ramirez, ${ }^{4}$ Helene F. Rosenberg, ${ }^{5}$ \\ Paul S. Foster, ${ }^{1-3}$ and Klaus I. Matthaei ${ }^{3}$ \\ ${ }^{1}$ Centre for Asthma and Respiratory Diseases (CARD), School of Biomedical Sciences, University of Newcastle, Newcastle, Australia; ${ }^{2}$ Vaccines, Immunity, \\ Viruses and Asthma Group, Hunter Medical Research Institute, Newcastle, Australia; ${ }^{3} J o h n$ Curtin School of Medical Research, Australian National University, \\ Canberra, Australia; ${ }^{4}$ Centre for Virus Research, School of Health Sciences, University of Canberra, Canberra, Australia; ${ }^{5}$ National Institute of Allergy \\ and Infectious Diseases, National Institutes of Health, Bethesda, MD
}

\begin{abstract}
Eosinophils are recruited to the lungs in response to respiratory syncytial virus (RSV) infection; however, their role in promoting antiviral host defense remains unclear. Here, we demonstrate that eosinophils express TLRs that recognize viral nucleic acids, are activated and degranulate after singlestranded RNA (ssRNA) stimulation of the TLR-7-MyD88 pathway, and provide host defense against RSV that is MyD88 dependent. In contrast to wild-type mice, virus clearance from lung tissue was more rapid in hypereosinophilic (interleukin-5 trans-
\end{abstract}

genic) mice. Transfer of wild-type but not MyD88-deficient eosinophils to the lungs of RSV-infected wild-type mice accelerated virus clearance and inhibited the development of airways hyperreactivity. Similar responses were observed when infected recipient mice were MyD88 deficient. Eosinophils isolated from infected hypereosinophilic MyD88-sufficient but not MyD88-deficient mice expressed greater amounts of IFN regulatory factor (IRF)-7 and eosinophil-associated ribonucleases EAR-1 and EAR-2. Hypereosinophilia in the airways of infected mice also correlated with increased expression of IRF-7, IFN- $\beta$, and NOS-2, and inhibition of NO production with the NOS-2 inhibitor LNMA partially reversed the accelerated virus clearance promoted by eosinophils. Collectively, our results demonstrate that eosinophils can protect against RSV in vivo, as they promote virus clearance and may thus limit virus-induced lung dysfunction. (Blood. 2007;110:1578-1586)

() 2007 by The American Society of Hematology

\section{Introduction}

There are numerous studies addressing the contribution of eosinophilic leukocytes to immune mechanisms regulating allergic diseases and granulomatous and fibrotic disorders, and eosinophil recruitment in response to helminthic parasite infection has been well documented. ${ }^{1}$ In contrast, there is only limited information on the role of eosinophils in immunity against infectious pathogens. Eosinophil recruitment was observed in response to acute severe respiratory syncytial virus (RSV) infection in human infants, ${ }^{2-4}$ and infection of airway epithelial cells results in the expression of several eosinophil chemoattractants. ${ }^{5,6}$ However, it is not at all clear whether eosinophils contribute to host defense or to the immunopathology observed in response to RSV infection. RSV infection is the most commonly identified cause of lower respiratory tract infections in infants, and severe disease has been associated with progression to childhood asthma, a disease state characterized by a mild to moderate eosinophilia. ${ }^{7,8}$ In addition, exacerbations of established asthma are most frequently caused by viral infections, of which RSV has been implicated. ${ }^{9}$

Recently, transcripts encoding Toll-like receptor (TLR)-1, TLR-4, TLR-7, TLR-9, and TLR-10, all of which coordinate innate and acquired immune responses, were shown to be expressed constitutively by eosinophils. ${ }^{10}$ Recognition of viral nucleic acids, including double-stranded RNA (dsRNA), single-stranded RNA (ssRNA), and dsDNA, occurs via activation of TLR-3, TLR-7, and TLR-9, respectively, and results in the production of type I IFNs and the initiation of the antiviral host response. ${ }^{11-15} \mathrm{RSV}$ is a negative ssRNA virus that generates dsRNA during its replication cycle. Although TLR-3 and TLR-7 both recognize viral RNA, TLR-3 uses toll-interleukin receptor domain-containing adaptorinducing IFN- $\beta$ (TRIF) and does not require MyD88, while TLR-7 signaling is MyD88 dependent. ${ }^{12}$ These differences in adaptor usage, along with the activation of different IFN regulatory factors (IRFs), are proposed to provide specificity in functional outcome. ${ }^{16}$ Although the molecular characterization of eosinophils (they also express MHC-I and MHC-II, antiviral ribonucleases, cytokines, and chemokines, and can engage T cells) supports the concept that this cell may contribute to the regulation of both innate and adaptive immunity, there is only limited direct evidence for a functional role of this cell in controlling viral infection. ${ }^{17-19}$

In this investigation, we sought to determine whether eosinophils contribute to RSV clearance through the expression of TLRs and MyD88-mediated mechanisms. We demonstrate that eosinophils express surface and intracellular TLRs associated with antiviral immunity and respond functionally to TLR ligands. Furthermore, by infecting the airways of hypereosinophilic (IL-5 $\mathrm{Tg}$ ) mice with RSV or by the adoptive transfer of MyD88sufficient, but not MyD88-deficient eosinophils to wild-type (WT) mice, we show that eosinophils mediate accelerated clearance via MyD88-dependent pathways, which also leads to suppression of RSV-induced pathology, including airways hyperreactivity (AHR).
Submitted January 30, 2007; accepted April 3, 2007. Prepublished online as Blood First Edition paper, May 10, 2007; DOI 10.1182/blood-2007-01-071340.
The publication costs of this article were defrayed in part by page charge payment. Therefore, and solely to indicate this fact, this article is hereby marked "advertisement" in accordance with 18 USC section 1734.

C 2007 by The American Society of Hematology 
Collectively, our data indicate that eosinophils contribute to antiviral immunity and play a beneficial role in limiting RSVinduced lung dysfunction.

\section{Materials and methods}

\section{Animals}

IL-5 $\mathrm{Tg}$ mice ${ }^{20}$ were crossed to the BALB/c genetic background for 10 generations (N10). MyD88-deficient mice (crossed to the BALB/c background for 7 generations [N7]; a generous gift from Dr Shizuo Akira, University of Osaka, Osaka, Japan) were crossed to the BALB/c background for a further 3 generations (N10) and then crossed with the N10.BALB/c IL-5 Tg mice (a generous gift from Dr Lindsay Dent, University of Adelaide, Adelaide, Australia) to generate IL-5 Tg-MyD88deficient mice as a source of MyD88-deficient eosinophils. N10.BALB/c dblGATA mice were a generous gift from Dr Alison Humbles and Dr C. Gerard (Harvard University, Boston, MA). Male mice were used at 6 to 8 weeks of age and treated in accordance to Australian National University Animal Welfare guidelines in a specific pathogen-free facility.

\section{Propagation of RSV}

RSV was propagated as previously described. ${ }^{21}$ Briefly, HEp- 2 cells were seeded in a T-150 flask. Cells were grown in the presence of Opti-MEM (Invitrogen, Mount Waverly, Australia) supplemented with 5\% HI-BS (Sigma, Castle Hill, Australia). Once the cells were confluent, they were infected with RSV at MOI 0.1 . The virus was allowed to adsorb for 1.5 hours at $37^{\circ} \mathrm{C} / 5 \% \mathrm{CO}_{2}$ before more media was added. When a $50 \%$ cytopathic effect was observed, the media was removed and replaced with PBS, and the flask was frozen and thawed twice. Lysed cells were scraped off and centrifuged to remove cell debris. Supernatant containing virus was further purified by centrifugation in a sucrose step gradient. The interphase on the top layer was collected and immediately transferred to sterile cryovials and stored at $-80^{\circ} \mathrm{C}\left(\right.$ at $\left.10^{6} \mathrm{TCID}_{50} / \mathrm{mL}\right)$.

\section{Virus titer determination}

Briefly, $1 \times 10^{6} \mathrm{HEp}-2$ cells $/ \mathrm{mL}$ were resuspended in media, and $100 \mu \mathrm{L}$ cell suspension were added to each well of a 96-well plate. Cells were incubated overnight at $37^{\circ} \mathrm{C} / 5 \% \mathrm{CO}_{2}$ or until $80 \%$ confluent. Lung tissues were homogenized, and serial dilutions were performed across the 96-well plate. Cells and virus were left to incubate for 1.5 hours to allow viral adsorption. After 5 days, the media was aspirated, and the wells were stained with $0.02 \%$ crystal violet and scored for the presence of syncytia. Results were entered into an automated spreadsheet of the Reed Muench formula, determining the tissue culture-infecting dose $\left(\mathrm{TCID}_{50}\right)$. Virus titer is presented as $\log _{10} \mathrm{TCID}_{50} / \mathrm{mL}$.

\section{Infection with RSV}

Mice were lightly anesthetized with isoflurane, laid flat upside-down, and inoculated via the intranasal route with RSV $\left(80 \times 10^{3} \mathrm{TCID}_{50} /\right.$ mouse $)$ in an $80-\mu \mathrm{L}$ volume.

\section{NO synthase inhibition in vivo}

$\mathrm{N}^{\omega}$-methyl-1-arginine (L-NMA; Sigma) or its inactive D-enantiomer (DNMA $)^{22}$ was administered $(5 \mathrm{mg} / 200 \mu \mathrm{L}$ PBS) via daily intraperitoneal injection. Viral titer was determined 3 days after inoculation.

\section{Eosinophil isolation and flow cytometry}

Spleens from IL-5 Tg or IL-5 Tg-MyD88-deficient mice were excised, and the splenocytes were released by teasing and gentle flushing with a syringe. Red blood cells were lysed with ACE. For flow cytometry assays, cells were preincubated with Fc $\gamma \mathrm{R}$ block in PBS/2\% FCS medium. Eosinophils were stained with a combination of Per-CP-conjugated Gr-1 and Alexa Fluor 647-conjugated CCR3 or PE-conjugated B220 and additionally identified by scatter properties. Analysis of cell-surface TLR expression was per- formed using anti-TLR-3 and anti-TLR-7 Ab (Imgenex, San Diego, CA), followed by detection with Alexa Fluor 488-goat anti-rabbit Ab $(1 \mu \mathrm{g} / \mathrm{mL})$. TLR-4 was detected directly with PE-conjugated Ab (eBioscience, San Diego, CA). For intracellular protein expression, cells were fixed with $4 \%$ paraformaldehyde for 5 minutes, then incubated in PBS/0.1\% saponin for 20 minutes followed by receptor detection as stated. For CD11b expression, cells were stained with PerCP-anti-CD11b 10 minutes after stimulation. Eosinophils incubated with an isotype-matched antibody with irrelevant specificity acted as a control. All Abs were purchased from BD Biosciences (North Ryde, Australia) unless stated. Samples were analyzed using a BD FACSort (Figures 2A,B, 3C) or BD CANTO (Figures 2C, 3D). Eosinophils recovered by FACS (BD Biosciences FACS Vantage Diva) were approximately $99 \%$ pure as determined by Giemsa-stained cytospin preparations.

\section{Quantitative RT-PCR}

RNA from sorted eosinophils $\left(>5 \times 10^{6}\right)$ was extracted using TRI Reagent (Ambion, Foster City, CA) following the manufacturer's protocol and stored at $-80^{\circ} \mathrm{C}$ in TE buffer. RNA integrity was assessed by agarose gel analysis. RNA was quantified using the Nanodrop spectrophotometer (Ambion), and $1 \mu \mathrm{g}$ was used for reverse transcription (RT) using random primers (SuperScript III, manufacturer's protocol, in a $20-\mu \mathrm{L}$ volume; Invitrogen). The cDNA volume was adjusted to $50 \mu \mathrm{L}$, and $5 \mu \mathrm{L}$ was used for real-time polymerase chain reaction (PCR) (ABI 7700; Applied Biosystems, Scoresby, Australia) using a reaction volume of $25 \mu \mathrm{L}$ (Platinum $2 \times$ Sybr mix; Invitrogen). Cycling conditions were $50^{\circ} \mathrm{C}$ for 2 minutes and $95^{\circ} \mathrm{C}$ for 2 minutes, followed by 40 cycles of $95^{\circ} \mathrm{C}$ for 15 seconds, $60^{\circ} \mathrm{C}$ for 30 seconds, and dissociation analysis. Relative expression values were determined by using serial dilutions of cDNA to establish a standard curve. Parallel reactions in which the reverse transcriptase enzyme was omitted showed minimal amplification. Expression of a housekeeping gene (HPRT) was also determined against a plasmid control, and relative gene expression was adjusted against expression of the housekeeping gene, which was not altered by RSV treatment. Primers designed and used were as follows. IRF-5: For-agatctacgaggtctgctccaacg, Rev-gccacttggtgtcttctttgggta; IRF-7: For-cttagccgggagcttggatctact, Revccettgtacatgatggtcacatcc; EAR-1: For-aatgctgttggtgtgtgtggaaat, Rev-cactggatacatgggactgtcctg; EAR-2: For-tctgtaacatcaccagtcggagga, Rev-cactggagctaaaatgtcccatcc; IFN- $\beta$ : For-agagttacactgcctttgccatcc, Rev-ccacgtcaatctttcctcttgctt; IFN- $\gamma$ : For-tcttgaaagacaatcaggccatca, Rev-gaatcagcagcgactcctttcc; and NOS-2: For-cctcggaggttcacctcactgt, Rev-cagaaacttcggaagggagcaat.

\section{Electron microscopy}

Eosinophils were isolated from spleens of IL-5 Tg mice and kept at $4^{\circ} \mathrm{C}$ at all times. Fluorescence-activated cell sorter (FACS)-purified eosinophils were incubated with medium or ssRNA/Lyo Vec (a 20-mer phosphothioate-protected ssRNA oligonucleotide containing a GU-rich sequence ${ }^{13}$ complexed with a cationic lipid; InvivoGen, San Diego, CA) for 10 minutes, then pelleted and fixed in $2 \%$ glutaraldehyde (EM Sciences, Hatfield, PA) in $0.1 \mathrm{M}$ sodium cacodylate buffer (ProSciTech, Thuringowa, Australia), $\mathrm{pH} 7.4$, at $4^{\circ} \mathrm{C}$ overnight. The samples were washed in $0.1 \mathrm{M}$ sodium cocadylate buffer and postfixed in $1 \%$ osmium tetroxide (ProSciTech) for 90 minutes, dehydrated in graded acetone solutions, and embedded in Spur resin (ProSciTech). Ultrathin sections (80-85 nm) were cut on a Reichert-Jung (Depew, NY) Ultracut E ultramicrotome. The sections were stained with $2 \%$ uranyl acetate (10 minutes) and lead citrate (3 minutes) before analysis using a Hitachi transmission electron microscope (H-7000; Hitachi, Tokyo, Japan).

\section{Detection of eosinophil peroxidase activity}

Mixed splenocytes were resuspended in PBS/2\% FCS, plated at 25000 eosinophils/well, and treated with $\mathrm{PBS} / 2 \%$ FCS or ssRNA $\left(1 \mu \mathrm{g} / \mathrm{mL}\right.$ ) for 20 minutes at $37^{\circ} \mathrm{C}$. The supernatant was collected, and eosinophil peroxidase (EPO) activity was measured as previously described. ${ }^{23}$ The product was measured at $490 \mathrm{~nm}$ using a colorimetric plate reader. Higher EPO content was indicated by higher units of optical density. Samples were performed in triplicate. 


\section{Adoptive transfer of eosinophils}

WT or MyD88-deficient mice were lightly anesthetized with alfaxalone (Vetoquinol, Buckingham, United Kingdom) via tail vein injection before intratracheal instillation (day 0 ) of $5 \times 10^{6}$ eosinophils in a $40-\mu \mathrm{L}$ volume or vehicle (PBS/2\% FCS). The trachea was visualized with a dissecting microscope, and a loosely inserted cannula was used to instill a suspension of WT or MyD88-deficient eosinophils. At 2 hours later, all treatment groups were inoculated (intranasal route) with RSV as described, and lung function was assessed at day 5, 24 hours prior to death (day 6).

\section{Measurement of AHR}

Responsiveness to methacholine (aerosolized from solutions containing 3.125-50 mg/mL; maximal effective response only shown) was assessed as enhanced Pause (Penh), measured in conscious, unrestrained mice using apparatus and software supplied by Buxco (Troy, NY). Measurement was performed as previously described. ${ }^{24}$ Notably, we have confirmed in the BALB/c strain that changes in Penh in response to methacholine directly correlate with changes in airway resistance to this spasmogen (data not shown). Thus, measuring changes in Penh reflects alterations in resistance and is indicative of enhanced airways responsiveness. Values are represented as Penh after methacholine minus baseline Penh.

\section{Characterization of mucus cell hyperplasia}

Lung tissue representing the central and peripheral airways were fixed in $10 \%$ phosphate-buffered formalin, sectioned, and stained with PAS and hematoxylin. Mucus-secreting goblet cells were expressed as cells/100 $\mu \mathrm{m}$ basement membrane (BM) length.

\section{Statistical analysis}

Data presented are the means plus or minus standard error ( \pm SEM). Datasets were analyzed by Student $t$ test.

\section{Results}

\section{Eosinophils promote viral clearance and limit RSV-induced mucus hypersecretion}

To examine the potential antiviral contributions of eosinophils to RSV infection, we inoculated BALB/c hypereosinophilic (IL-5 Tg) and WT mice with $80 \times 10^{3} \mathrm{TCID}_{50}$ of RSV via the intranasal route and compared the rate of viral clearance by a syncytia-detection assay on days 1, 3, 5, and 8 after inoculation (dpi). IL-5 Tg mice express IL-5 constitutively from $\mathrm{T}$ cells and are characterized by a blood eosinophilia and accumulation of eosinophils in the spleen (approximately 30\% eosinophils). The lungs of IL-5 Tg hypereosinophilic mice are characterized by a mild eosinophilia and goblet cell hyperplasia, but display normal lung reactivity to methacholine. ${ }^{25}$ Beginning at $3 \mathrm{dpi}$, the lungs of the hypereosinophilic mice contained significantly fewer virions compared with the WT mice (Figure 1A). RSV infection of the hypereosinophilic mice resulted in recruitment of eosinophils and neutrophils into the airways from 1 dpi (data not shown). The eosinophilic response was greater in hypereosinophilic mice than in WT mice, although the numbers of neutrophilic infiltrates were similar between these strains (data not shown). RSV infection resulted in an increase in the number of goblet cells secreting mucus into the airways in both hypereosinophilic and WT mice within 24 hours (data not shown). In the WT mice, the number of goblet cells was further increased at $5 \mathrm{dpi}$, while in the hypereosinophilic mice, this tissue tropism was resolving (Figure 1B). Indeed, at $5 \mathrm{dpi}$ and $8 \mathrm{dpi}$, RSV-induced increases in goblet cells were significantly higher in WT mice compared with hypereosinophilic mice.
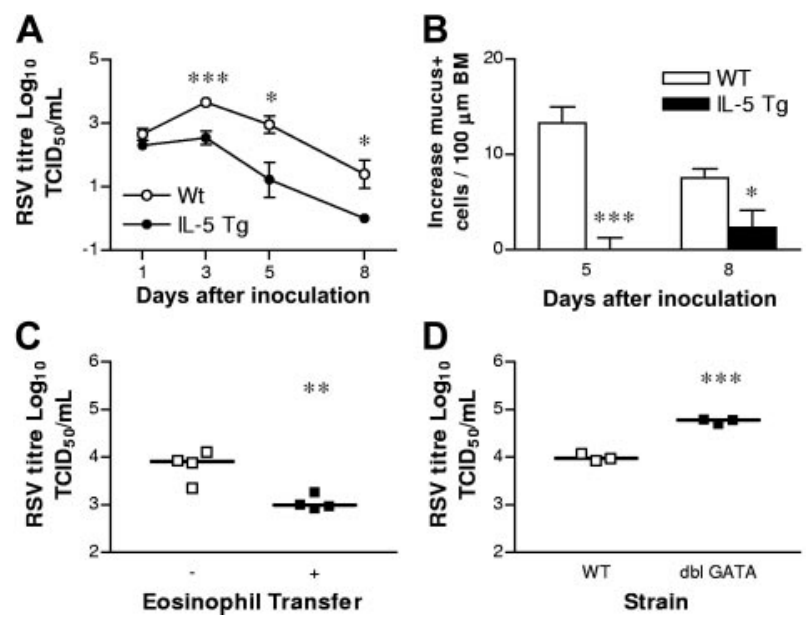

Figure 1. Hypereosinophilic mice show accelerated viral clearance in the lung (A) WT (O) and hypereosinophilic (IL-5 Tg; $\bullet$ ) mice were inoculated with RSV at day 0 and viral clearance or (B) increases in numbers of mucus-secreting cells per $100 \mu \mathrm{m}$ of airway epithelial basement membrane monitored at the times indicated. (C) Eosinophils $\left(5 \times 10^{6}\right.$ cells) isolated from hypereosinophilic mice were adoptively transferred to WT mice 2 hours prior to inoculation with RSV. Viral titer was determined at 6 dpi. Bars represent the median value. (D) WT or $\triangle$ dbIGATA mice were inoculated with RSV and viral titer quantified at 5 dpi. Data represent the mean plus or minus SEM ( $n=3-6$ mice per experimental group). Bars represent the median value. ${ }^{\star} P<.05 ;{ }^{\star \star} P<0.01 ;{ }^{* \star *} P<.001$.

To confirm that accelerated virus clearance was due specifically to eosinophils and not the overexpression of IL-5, we FACSpurified eosinophils ( $>98 \%$ pure) from the spleens of hypereosinophilic mice, and performed an adoptive transfer directly into the lungs of WT mice 2 hours before RSV inoculation. Eosinophils directly transferred into the airways have been shown to remain functional and can migrate into the lung and local pulmonary lymph nodes, where they actively participate in immune responses. ${ }^{26,27}$ The transfer of donor eosinophils significantly enhanced viral clearance compared with vehicle-treated controls at 5 dpi (Figure 1C). To demonstrate that eosinophils confer protection from RSV, we used $\Delta$ dblGATA mice, whose eosinophil lineage is selectively and severely disrupted. ${ }^{28,29}$ The clearance of RSV was significantly diminished in $\triangle$ dblGATA mice compared with WT controls (Figure 1D).

\section{Eosinophils express surface and intracellular toll-like receptors}

As human eosinophils constitutively express transcripts encoding TLR-1, TLR-4, TLR-7, TLR-9, and TLR-10, ${ }^{10}$ we speculated that they may use pattern recognition receptors (PRR) to promote antiviral host defense. To determine whether murine eosinophils expressed TLRs implicated in the recognition of RSV, we explored TLR-3, TLR-4, and TLR-7 surface and intracellular protein expression on eosinophils isolated from the spleens of naive (uninfected) hypereosinophilic mice by flow cytometry. These TLRs were predominantly expressed intracellularly, with only low levels detected on the cell surface (Figure 2A). The signal intensity observed for surface expression of TLRs was only significantly different from isotype-matched control antibody for TLR-7 (Figure $2 \mathrm{~B} ; P<.01$ ), while both the expression of intracellular TLR-3 and TLR-7 were both significantly different than isotype control (Figure 2B; $P<.01$ ). The expression of TLR-4 was greater than the isotype control in all samples tested, but did not reach statistical significance. We also observed that TLR-5 was expressed on the surface and intracellularly within eosinophils (data not shown).

Next, we analyzed TLR-7 expression on eosinophils and neutrophils isolated from lungs of naive hypereosinophilic mice. Eosinophils in the 
A
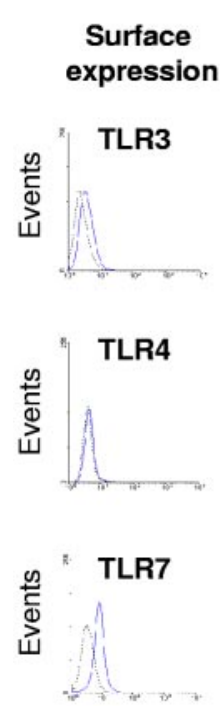

B

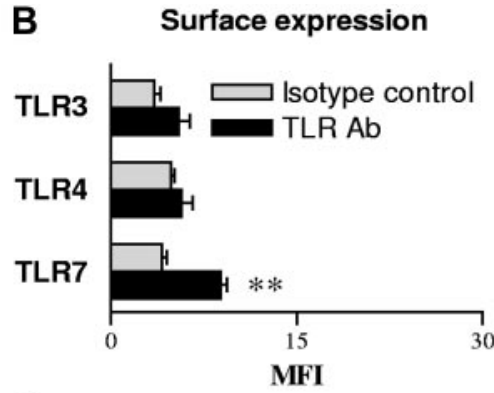

Intracellular expression

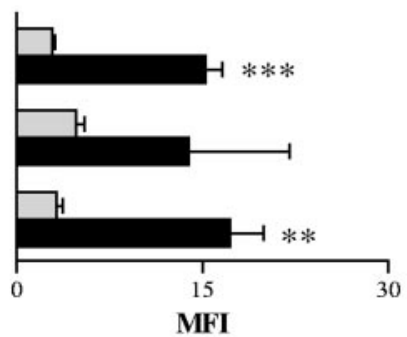

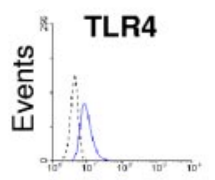

C
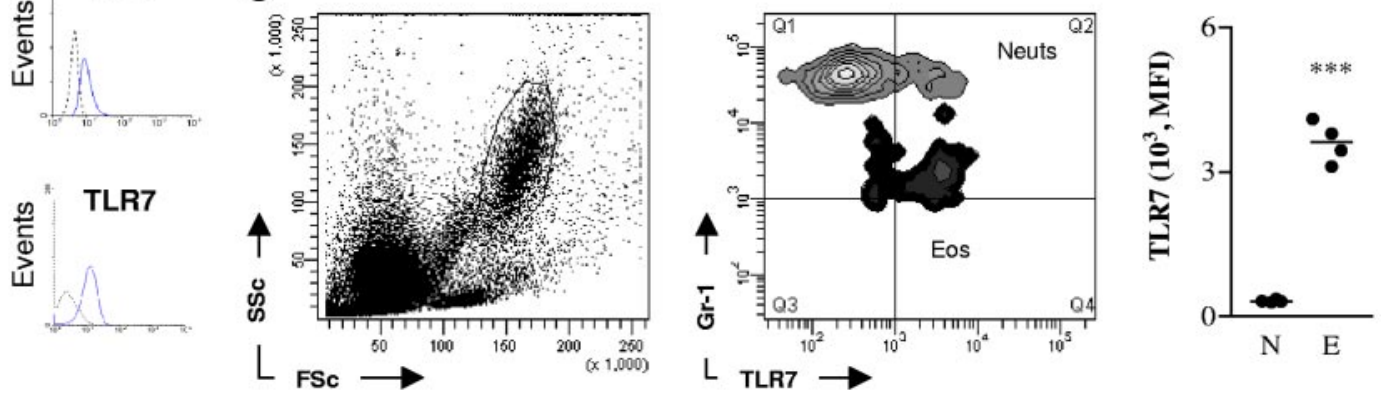

Figure 2. Eosinophils express surface and intracellular toll-like receptors. (A) Eosinophils from the spleens of hypereosinophilic mice were identified by CCR3 expression and high side scatter and stained for TLR-3, TLR-4, and TLR-7. Dashed lines denote isotype control (and were similar to unstained cells); solid lines represent respective TLR antibody. To detect intracellular TLR expression, splenocytes were pretreated with saponin. (B) Summary of mean fluorescence intensity (MFI) of surface and intracellular TLR expression by splenic eosinophils. The mean of 3 independent experiments is shown. (C) Neutrophils (Neuts) and eosinophils (Eos) were identified in the lungs of hypereosinophilic mice by scatter properties (left panel) and Gr-1 expression (middle panel). Comparative MFI of TLR-7 expression on neutrophils (N) and eosinophils (E) is shown in the right panel. Data represent the mean plus or minus SEM $\left(n=3-8\right.$ mice per experimental group). ${ }^{\star} P<.05 ;{ }^{* \star} P<.01 ;{ }^{\star \star \star} P<.001$.

lungs were found to be TLR- $7^{+}$, and the intensity of this signal was 10-fold greater than that of lung neutrophils (Figure 2C). The expression of TLR-7 was similar between eosinophils derived from the lungs or spleen, allowing us to isolate cells in greater numbers from the spleen for characterization of TLR-7 function.

\section{The TLR-7 ligand ssRNA activates eosinophils}

To support a role for TLR-7 in eosinophil activation, we measured the effect of the TLR-7 ligand, ssRNA, on cell functions that may contribute to antiviral immunity. First, we used an assay that uses the peroxidase-sensitive substrate o-phenylenediamine as a marker of EPO release. ${ }^{30}$ Treatment of eosinophil-rich splenocytes from hypereosinophilic mice with ssRNA for 10 minutes resulted in a significant increase in the amount of EPO detected in culture supernatants (Figure 3A; $P<.01$ ). In other experiments, we observed EPO release within 2 minutes of stimulation (data not shown). We concluded that most of the EPO released was derived from eosinophils ( $>30 \%$ of the total population) in these cultures, as splenocyte populations from hypereosinophilic mice possessed extremely low numbers of neutrophils $(<3 \%)$; similarly, the assay design, which uses the formation of bromide derivatives, preferentially detects EPO as opposed to neutrophil myeloperoxidase. To demonstrate TLR-7-mediated eosinophil degranulation directly, we also evaluated ultrastructural alterations occurring within the granules of eosinophils after treatment with ssRNA by electron microscopy. FACS-purified eosinophils were treated for 10 minutes with ssRNA or medium and then fixed and processed for visualization. Untreated eosinophils isolated from the spleens of hypereosinophilic mice contained granules that were evenly distributed throughout the cytoplasm and appeared not to be activated (Figure $3 \mathrm{Bi}, \mathrm{ii})$. By contrast, after treatment with ssRNA, the granules clustered in one area of the cells (Figure 3Biii), and the granules either had lost their crystal core or had become inverted (Figure 3Biv; arrows), indicating activation and piecemeal degranulation.
We next investigated the effect of ssRNA on expression of $\mathrm{CD} 11 \mathrm{~b}$ as a marker of cell activation for phagocytosis. ${ }^{31}$ Extending earlier work by Nagase et al, ${ }^{10}$ we found that TLR-7 activation increased eosinophil cell-surface expression of CD11b within minutes of treatment (Figure 3C). Finally, we isolated eosinophils from hypereosinophilic MyD88-deficient mice to determine whether increased CD11b expression was mediated through the toll IL-1 receptor resistance (TIR) domain adaptor protein, MyD88. ssRNAinduced CD11b expression was clearly reduced in MyD88deficient eosinophils (Figure 3D).

\section{Eosinophils mediate viral clearance through the TIR adaptor protein MyD88}

To provide further direct evidence in support of a role for eosinophil TLR signaling in the clearance of RSV, we used recipient WT or MyD88-deficient mice and transferred MyD88-sufficient (WT) or MyD88-deficient eosinophils to the lung 2 hours prior to RSV inoculation (Figure 4A). At first, we demonstrated that RSV clearance was significantly delayed in MyD88-deficient mice compared with WT mice (data not shown). The subsequent transfer of MyD88-sufficient eosinophils to WT mice markedly decreased viral titer at 5 dpi (Figures 1C, 4B). However, there was no significant difference in viral clearance between infected WT mice and those that received MyD88-deficient eosinophils (Figure 4B). An identical pattern emerged when MyD88deficient mice were the recipients of WT eosinophils (Figure 4C). The transfer of MyD88-sufficient eosinophils resulted in a decreased viral load in both WT and MyD88-deficient mice, and the absence of MyD88 rendered the eosinophils ineffective.

\section{Eosinophil transfer reduces AHR in RSV-infected mice}

Because RSV infection has been associated with AHR, we next examined whether eosinophil-mediated viral clearance could suppress RSV-induced AHR. Consistent with the findings of others, 
A
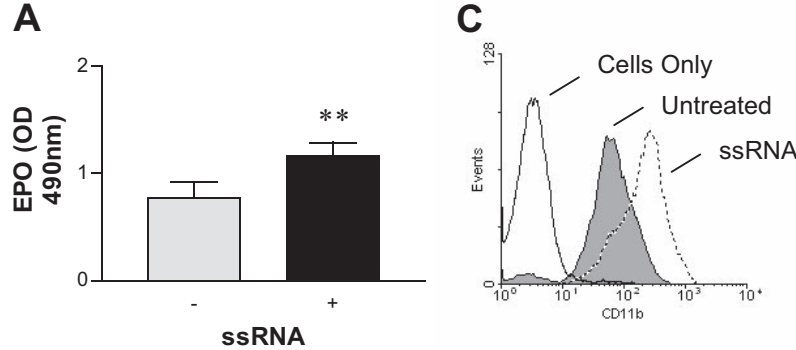

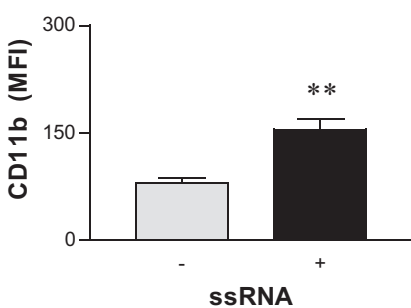

D

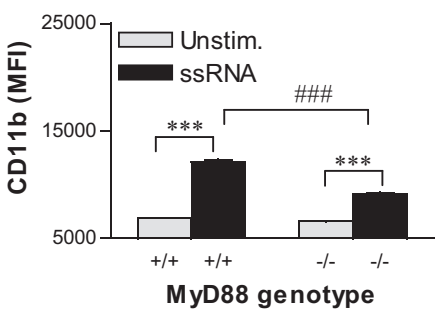

Figure 3. ssRNA-induced activation of eosinophils. (A) Isolated splenocytes from hypereosinophilic mice were activated with ssRNA for 10 minutes, and peroxidase activity was measured to determine EPO release. (B) FACS-purified eosinophils were stimulated with medium (Bi-Bii) or ssRNA (1 $\mu \mathrm{g} /$ $\mathrm{mL}$ ) (Biii, Biv) for 10 minutes, then changes in ultrastructural morphology were examined by electron microscopy. Arrows demarcate secondary granules, with irregular or faded electron-dense cores documenting degranulation in a representative micrograph. Scale bar equals $1 \mu \mathrm{M}$ (left panels) and $2 \mu \mathrm{M}$ (right panels) see "Materials and methods, Electron microscopy" for detailed image acquisition information. (C) Isolated mixed splenocytes from hypereosinophilic mice were treated with SSRNA for 10 minutes, then stained for CD11b expression. A representative histogram gated on eosinophils is presented in the left panel. The MFI of CD11b expression is shown in the right panel. These data were collected on a FACSort. (D) Eosinophils were isolated from hypereosinophilic MyD88-sufficient or -deficient mice and treated with ssRNA $(1 \mu \mathrm{g} / \mathrm{mL})$ for $10 \mathrm{~min}$ utes, then stained for CD11b expression. These data were collected on a BD CANTO. Data represent the mean plus or minus SEM ( $n=3-8$ mice per experimental group) ${ }^{\star \star} P<.01 ;{ }^{* \star *} P<.001 ; \# \# \# P<.001$.

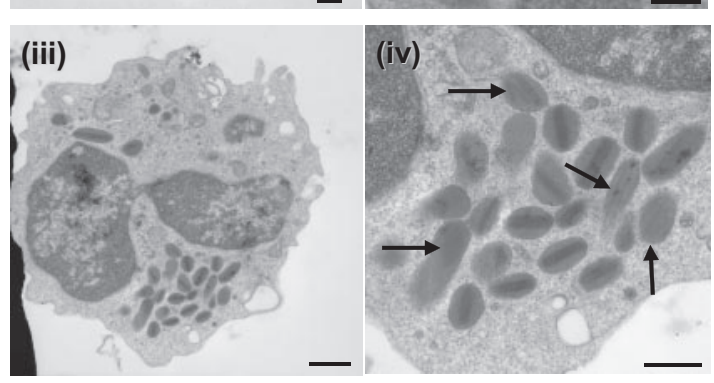

inoculation with RSV induced AHR to methacholine (Figure 4D); in naive mice, $50 \mathrm{mg} / \mathrm{mL}$ methacholine induced a mean plus or minus SEM increase of 1.09 plus or minus 0.29 penh; $n=4$. Values measured in naive mice were significantly lower than those in RSV-inoculated mice). ${ }^{32,33}$ The transfer of WT eosinophils significantly reduced the magnitude of AHR to methacholine (Figure 4D). In contrast, the transfer of MyD88-deficient eosinophils did not inhibit the development of RSV-induced AHR. Similarly, the transfer of MyD88-sufficient eosinophils to MyD88deficient recipients suppressed RSV-induced AHR, while eosinophils deficient for MyD88 were without effect (Figure 4E).

\section{Effect of RSV infection on eosinophil gene expression}

Intranasal inoculation with RSV resulted in the increased expression of surface CD11b on eosinophils in the spleens of hypereosinophilic mice (data not shown). Having demonstrated that RSV induces systemic effects, we next determined if antiviral genes were induced in eosinophils in response to infection. We FACSpurified splenic eosinophils from mice at $1 \mathrm{dpi}$ and determined the expression of transcripts encoding IRF-5 and IRF-7, both signaling components required for effective TLR-7 signaling. ${ }^{34}$ Eosinophils expressed both IRF-5 and IRF-7 at baseline. However, only IRF-7 expression was significantly induced ( $>7$-fold) in response to $\mathrm{RSV}$, and this effect was dependent on MyD88 (Figure 5A).

We have previously proposed that eosinophils mediate host defense from ssRNA viruses, at least in part, through the expression of potent ribonucleases. ${ }^{17,19}$ Here, we sought to determine whether RSV challenge resulted in the increased expression of mouse eosinophil-associated ribonucleases, the rodent orthologs of eosinophil-derived neurotoxin and eosinophil cationic protein in primates. ${ }^{19}$ Within 24 hours of inoculation, both mEAR-1 and mEAR-2 were significantly induced (Figure 5A), and this effect was MyD88 dependent. We next determined whether RSV altered the transcription of genes encoding type I or type II IFNs in eosinophils. Transcripts encoding both IFN- $\beta$ and IFN- $\gamma$ were detected in eosinophils, but neither gene was induced at the early timepoint studied after RSV infection (1 dpi). Collectively, these data indicate that eosinophil TLR signaling plays a critical role in gene regulation in eosinophils and in the recognition of RSV.

We next analyzed the temporal expression of IRF-7, IFN- $\gamma$, and IFN- $\beta$ in the lungs of RSV-infected hypereosinophilic and WT mice (Figure 5B). Transcript encoding IRF-7 was significantly increased in hypereosinophilic mice within 24 hours, peaked at 3 days, and remained significantly elevated compared with that of WT mice at $8 \mathrm{dpi}$. Similarly, IFN- $\gamma$ expression was significantly increased in hypereosinophilic mice compared with that of WT mice from 1 dpi (data not shown). IFN- $\beta$ gene expression also peaked at $3 \mathrm{dpi}$, but in contrast to IRF-7, the onset of expression was delayed until 3 dpi, when expression was significantly elevated in the hypereosinophilic mice compared with that of RSV-infected WT mice.

\section{Eosinophil NO production contributes to antiviral immunity from RSV}

Nitric oxide (NO) has been implicated in antiviral host defense and may also be functionally protective through bronchodilatory mechanisms. To determine whether NO contributed to eosinophilmediated antiviral immunity, we first examined the temporal profile of NOS-2 expression in the lungs of WT and hypereosinophilic mice in response to RSV. The expression of NOS-2 increased in the lungs of both strains of mice (Figure 5B). However, the lungs of the 

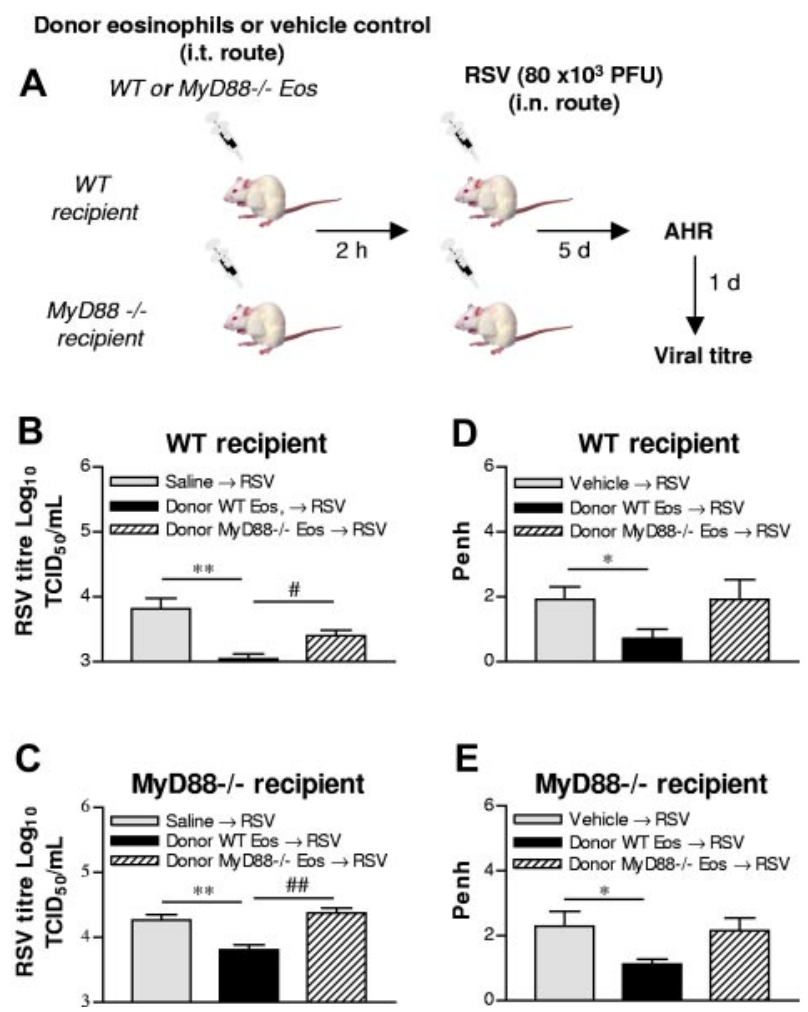

Figure 4. Adoptive transfer of WT but not MyD88-deficient eosinophils significantly accelerates viral clearance and suppresses RSV-induced AHR. (A) Experimental design. $(B, C)$ Eosinophils were isolated from the spleens of hypereosinophilic MyD88-sufficient or -deficient mice and sorted by FACS. WT or MyD88-1- eosinophils were instilled directly into the trachea 2 hours before inoculation with RSV into WT (B) or MyD88-deficient (C) mice, and virus clearance was assessed at 6 dpi $(D, E)$. Mice were treated as described in panels $B, C$ and assessed for alterations of airway responsiveness to methacholine $(50 \mathrm{mg} / \mathrm{mL}$, dose that gives maximal responsiveness) by whole-body plethysmography. Data represent the mean plus or minus SEM ( $\mathrm{n}=3-4$ mice per experimental group). ${ }^{*} P<.05$; ${ }^{\star \star} P<.01 ; \# P<.05 ; \# \#<.01$ hypereosinophilic mice contained significantly higher expression of NOS-2 compared with the WT mice at 3 dpi (Figure 5B).

To establish the functional significance of the NOS-2 expression and potential contribution of $\mathrm{NO}$ to the accelerated clearance observed in hypereosinophilic mice (Figure 1A), WT and hypereosinophilic mice were treated to the NOS-2 inhibitor L-NMA or its inactive D-NMA prior to and during infection. L-NMA significantly delayed the clearance of RSV in WT mice, and completely reversed the advantage conferred by the hypereosinophilic status of IL-5 Tg mice (Figure 5C).

\section{Discussion}

Eosinophil recruitment and degranulation in lung tissue has been observed in response to RSV infection in humans; $;^{2-4,35,36}$ however, the contributions of this cell to disease and innate immunity have not been clearly defined. We demonstrate here that an airway eosinophilia present constitutively in hypereosinophilic (IL-5 Tg) mice or introduced by transfer of eosinophils directly to the lungs, results in the accelerated clearance of RSV virions and reduction in associated airway dysfunction, including mucus hypersecretion and AHR. We demonstrate that ssRNA, the ligand for TLR-7, induces functional activation of eosinophils, specifically degranulation and increased expression of the phagocytic receptor CD11b. Mechanistically, we show that eosinophil-mediated accelerated RSV clearance and attenuated lung dysfunction are dependent on the TIR adaptor molecule MyD88 and the production of NO by NOS-2.

TLRs are a conserved family of PRRs that bridge innate pathogen recognition and the development of adaptive ( $\mathrm{T}$ and $\mathrm{B}$ lymphocyte) immune responses. The immediate response to RSV infection in immunocompetent hosts includes recruitment of proinflammatory granulocytes, which themselves express TLRs. A study by Nagase et al $^{10}$ demonstrated that human neutrophils and eosinophils constitutively express transcripts encoding most known TLRs, including TLR-7, which was subsequently found to recognize ssRNA. ${ }^{12-14}$ Notably, stimulation of eosinophils with the synthetic TLR-7 ligand R-848 led to the generation of reactive genes, and the inhibition of NOS-2 abrogates the protection conferred by eosinophils. (A) FACS-purified splenic eosinophils were isolated from naive hypereosinophilic mice (畨) or hypereosinophilic ( $\square$ ) and hypereosinophilic MyD88-deficient (四) mice at $1 \mathrm{dpi}$, and expression of indicated genes were analyzed by quantitative RT-PCR. All quantitative assessments were normalized to $H P R T$, and fold induction was calculated relative to naive hypereosinophilic mice. ${ }^{*}$ Comparison between naive and RSV inoculated hypereosinophilic mice. \#Comparison between RSVinoculated hypereosinophilic and hypereosinophilic MyD88 ${ }^{-1}$ mice. (B) WT $(\bigcirc)$ and hypereosinophilic $(\bullet)$ mice were inoculated, lung samples were collected at the indicated times after infection, and expression of indicated genes were analyzed by quantitative RT-PCR. (C) WT and hypereosinophilic mice were pretreated with L-NMA ( $\square$ ) or D-NMA (圆) prior to inoculation with RSV. Lung viral titer was measured at $3 \mathrm{dpi}(\mathrm{n}=3-6$ mice $) .{ }^{*} P<.05$; \#P<.05; ${ }^{\star \star} P<.01 ; \# \# P<.01 ;{ }^{\star \star \star} P<.001 ; \# \# \#<.001$. Error bars are SEM.
A

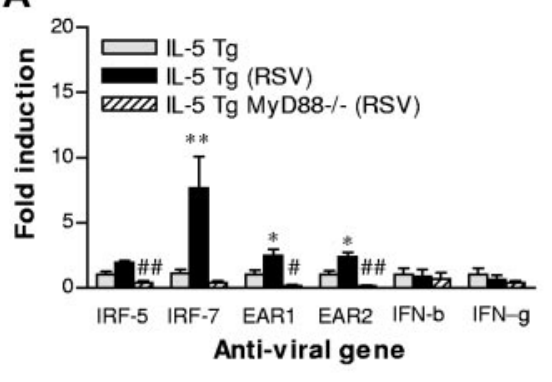

B

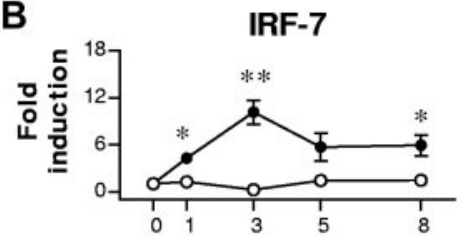

IFN-b

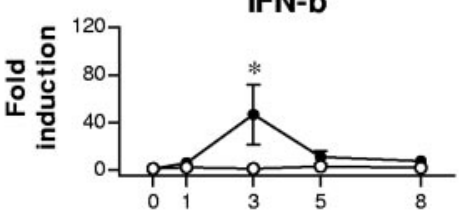

NOS-2
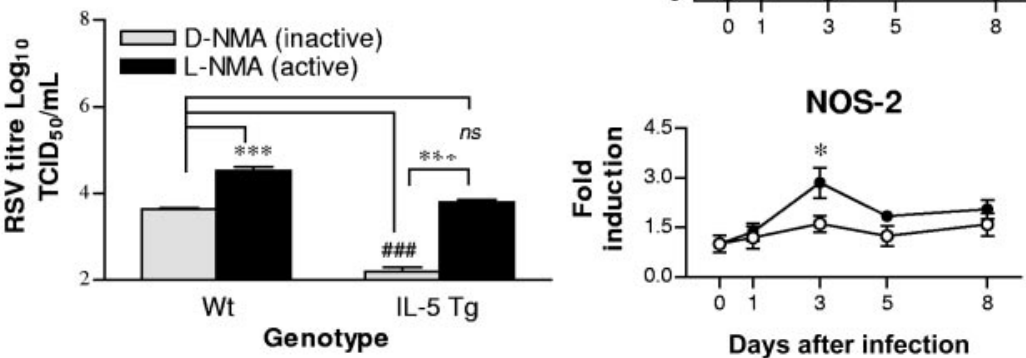
oxygen species, findings which suggested to us that eosinophils may contribute to host defense against viral infection via this PRR. A study by Adamko et al $^{18}$ demonstrated that the clearance of parainfluenza virus in guinea pigs was advanced by prior sensitization to ovalbumin and the generation of T helper $2(\mathrm{Th} 2)$-mediated pulmonary eosinophilia, an effect that was reversed when eosinophils were depleted with monoclonal anti-IL-5. ${ }^{18} \mathrm{We}$ also have previously demonstrated in vitro that eosinophils limit virus infectivity through the expression of eosinophil-associated ribonucleases. ${ }^{17,19}$ Here, we extend these findings by demonstrating that hypereosinophilic mice undergo accelerated clearance of RSV, while eosinophil-deficient mice demonstrate delayed clearance. Notably, while the transfer of WT eosinophils to infected WT mice also promoted accelerated RSV clearance, the transfer of MyD88deficient eosinophils did not function effectively in this fashion. Although MyD88 is 1 of 5 identified TIR adaptor proteins, ${ }^{16}$ all known TLRs with the exception of TLR-3 use this adaptor for signaling. Thus, although it is unlikely that the engagement of TLR-3 mediates the protective effect conferred by donor WT eosinophils in this particular setting, we cannot rule out a role for this PRR in RSV disease in general. ${ }^{37}$ In the mouse, we demonstrate at the protein level that eosinophils express another TLR that can recognize molecular patterns expressed by RSV, namely TLR-7.

Extending earlier work by Nagase et $\mathrm{al}^{10}$ we found that TLR-7 activation significantly increased expression of CD11b in eosinophils. These results are also consistent with observations that eosinophils from infants with lower respiratory tract RSV disease demonstrated increased expression of CD11b. ${ }^{38}$ Although ssRNA-induced CD11b expression was significantly reduced when MyD88-deficient eosinophils were used, the response was not entirely ablated. ${ }^{39}$ These data suggest that ssRNA may operate through another as-yet unidentified recognition receptor, perhaps located in the cytosol and similar to those activated by dsRNA. ${ }^{40}$

The IRF family of transcription factors mediates a number of functional outcomes downstream of TLR activation, and have recently been demonstrated as central mediators of TLR-7 signaling. ${ }^{34}$ IRF-7 interacts with MyD88 and is essential for the inductionof type I IFNs. ${ }^{41}$ In addition, IRF- 5 has been identified as a central mediator of TLR-7 signaling. ${ }^{34}$ RSV inoculation increased the expression of IRF-7 (7-fold) in a MyD88-dependent manner. This is the first study to show definitively that IRF-7 expression by eosinophils increases rapidly in response to viral infection in vivo. The induced expression of IRF-7 may have been more pronounced since this transcription factor, in contrast to IRF-5, is expressed in relatively small amounts in most cells. ${ }^{42,43}$ The marked increase in IRF-7 in the lungs of hypereosinophilic mice following inoculation with RSV preceded a pronounced increase in expression of IFN- $\beta$. Importantly, the production by and activation of epithelial cells by type I IFNs has been demonstrated to initiate apoptosis as a protective mechanism to limit viral replication. It is intriguing to note that epithelial cells obtained from people with asthma have impaired production of IFN- $\beta$ and IFN- $\lambda$ in association with increased virus replication. ${ }^{44,45}$ Our findings indicate that, in response to RSV, the signaling mechanisms of eosinophils are primed for activation and induction of host defense mechanisms promoting increased production of IFN- $\beta$. These findings suggest an important role for eosinophils in promoting host defense against RSV.

We have previously demonstrated that the ability of eosinophils to limit virus infectivity in vitro was suppressed through the addition of a placental ribonuclease inhibitor, suggesting that eosinophils may mediate their activity, at least in part, through the expression and release of potent ribonucleases. ${ }^{17,19}$ Within 24 hours of inoculation, we observed increased expression of transcripts encoding both mEAR-1 and mEAR-2 in airway eosinophils. This effect was not observed in eosinophils isolated from MyD88deficient hypereosinophilic mice, indicating that expression of these genes was dependent on TLR activation, findings which implicate either TLR-4 or TLR-7 but not TLR-3 in the recognition of RSV in vivo.

Two studies have demonstrated that TLR-4-deficient mice are immunocompromised and show delayed clearance of RSV compared with WT mice, ${ }^{21,46}$ although the interpretation of the results has been questioned, ${ }^{47}$ and TLR-4 had no impact on virus clearance in the natural mouse pneuomovirus infection model. ${ }^{48}$ Since both TLR-4 and TLR-7 use MyD88, we chose to determine whether eosinophils mediate protective immunity in vivo through MyD88-dependent signaling pathways. Although the transfer of WT eosinophils accelerated RSV clearance, donor MyD88-deficient eosinophils were without effect. Accelerated virus clearance occurred in response to WT donor eosinophils regardless of whether the recipient mouse was WT or MyD88-deficient, implying that eosinophil MyD88 pathways are necessary and sufficient for the accelerated viral clearance mediated by this cell.

Intriguingly, we also observed that eosinophil-mediated clearance of RSV corresponded with a significant reduction in the magnitude of airway reactivity to methacholine provocation. In the setting of allergic asthma, where eosinophils are prevalent in the airway mucosa and exacerbations of disease are most frequently caused by viral infections, eosinophils have often been implicated as a causative agent of AHR. ${ }^{49-51}$ However, the contribution of eosinophils to AHR in both man and mouse remains controversial. ${ }^{52,53}$ Moreover, in mouse models of asthma in which eosinophils contribute to AHR, they have been shown to do so by promoting the release of IL-13 from allergen-specific $\mathrm{CD}^{+}{ }^{+} \mathrm{Th} 2$ cells, an allergic inflammatory mechanism that is not functional in RSV-challenged mice. ${ }^{54}$ Furthermore, it is likely that the role ofeosinophils in the modulation of immune or pathologic processes is highly dependent on signals received from the microenvironment and signals that are associated with specific inflammatory responses. Collectively, our data demonstrate that eosinophils are able to recognize RSV-associated molecular patterns in a TLR-MyD88-dependent manner and to orchestrate an innate antiviral host response to RSV.

The induction of NOS-2 by inflammatory cells and by pulmonary epithelial cells following infection with RSV is well documented. ${ }^{55}$ Indeed, we have previously demonstrated in vitro that the production of NO from epithelial cells infected with RSV correlates inversely with viral titer, an effect that was reversed by the NOS inhibitor NG-methyl-L-arginine. ${ }^{56} \mathrm{Re}$ cently, Stark et $\mathrm{al}^{57}$ demonstrated that in vivo inhibition of NOS-2 during RSV infection resulted in increased RSV titers in the lungs. We extended these studies to demonstrate that RSV challenge of hyperesoinophilic mice results in a significant induction of NOS-2 in the lungs of these mice. Moreover, we show that inhibition of NOS-2 with L-NMA attenuated the accelerated viral clearance, suggesting that eosinophil-derived NO contributes to innate protection against RSV.

In summary, our findings support a role for the eosinophil in innate antiviral immunity. Specifically, we demonstrate that the eosinophil uses TLR-dependent pathways, in particular TLR-7 and 
its downstream signaling molecule MyD88, to promote host defense against RSV.

\section{Acknowledgments}

The authors would like to thank Di Gooding and Wayne Damcevski for assistance in the generation of IL-5 TgMyD88-1- mice.

This work was supported by National Health and Medical Research (NHMRC) Program grant (224207). S.P. is a recipient of an International Fellowship from the The Royal Society. S.M. is a recipient of an NHMRC R. Douglas Wright Fellowship.

\section{Authorship}

Contribution: S.P. designed the study and co-wrote the paper with H.F.R, P.S.F and K.I.M. S.P., C.E.L., S.M., M.N., R.R., and K.I.M performed research.

The laboratories of P.S.F. and K.I.M. contributed equally to this work.

Conflict-of-interest disclosure: The authors declare no competing financial interests.

Correspondence: Paul Foster, Centre for Asthma and Respiratory Diseases, School of Biomedical Sciences, David Maddison Clinical Sciences Building, Watt St, Newcastle, NSW, 2300 Australia; e-mail: paul.foster@newcastle.edu.au.

\section{References}

1. Rothenberg ME, Hogan SP. The eosinophil. Annu Rev Immunol. 2006;24:147-174.

2. Garofalo R, Kimpen JL, Welliver RC, Ogra PL. Eosinophil degranulation in the respiratory tract during naturally acquired respiratory syncytial virus infection. J Pediatr. 1992;120:28-32.

3. Everard ML, Swarbrick A, Wrightham M, et al. Analysis of cells obtained by bronchial lavage of infants with respiratory syncytial virus infection. Arch Dis Child. 1994;71:428-432.

4. Harrison AM, Bonville CA, Rosenberg HF, Domachowske JB. Respiratory syncytical virus-induced chemokine expression in the lower airways: eosinophil recruitment and degranulation. Am J Respir Crit Care. Med 1999;159:1918-1924.

5. Matthews SP, Tregoning JS, Coyle AJ, Hussell T, Openshaw PJ. Role of CCL11 in eosinophilic lung disease during respiratory syncytial virus infection. J Virol. 2005;79:2050-2057.

6. Johnson TR, Parker RA, Johnson JE, Graham BS. IL-13 is sufficient for respiratory syncytial virus $\mathrm{G}$ glycoprotein-induced eosinophilia after respiratory syncytial virus challenge. J Immunol. 2003; 170:2037-2045.

7. Singh AM, Moore PE, Gern JE, Lemanske RF Jr, Hartert TV. Bronchiolitis to asthma: a review and call for studies of gene-viral interactions in asthma causation. Am J Respir Crit Care Med. 2006;19:19.

8. Everard ML. The role of the respiratory syncytial virus in airway syndromes in childhood. Curr Allergy Asthma Rep. 2006;6:97-102.

9. Busse WW, Gern JE. Viruses in asthma. J Allergy Clin Immunol. 1997;100:147-150.

10. Nagase $\mathrm{H}$, Okugawa $\mathrm{S}$, Ota $\mathrm{Y}$, et al. Expression and function of Toll-like receptors in eosinophils: activation by Toll-like receptor 7 ligand. J Immunol. 2003;171:3977-3982.

11. Alexopoulou L, Holt AC, Medzhitov R, Flavell RA. Recognition of double-stranded RNA and activation of NF-kappaB by Toll-like receptor 3. Nature. 2001;413:732-738.

12. Diebold SS, Kaisho T, Hemmi H, Akira S, Reis e Sousa C. Innate antiviral responses by means of TLR7-mediated recognition of single-stranded RNA. Science. 2004;303:1529-1531.

13. Heil $F$, Hemmi $H$, Hochrein $H$, et al. Species-specific recognition of single-stranded RNA via tolllike receptor 7 and 8 . Science. 2004;303:15261529.

14. Lund JM, Alexopoulou L, Sato A, et al. Recognition of single-stranded RNA viruses by Toll-like receptor 7. Proc Natl Acad Sci U S A. 2004;101: 5598-5603.

15. Lund J, Sato A, Akira S, Medzhitov R, Iwasaki A Toll-like receptor 9-mediated recognition of Herpes simplex virus- 2 by plasmacytoid dendritic cells. J Exp Med. 2003;198:513-520.
16. O'Neill LA, Fitzgerald KA, Bowie AG. The Toll-IL-1 receptor adaptor family grows to five members. Trends Immunol. 2003;24:286-290.

17. Domachowske JB, Dyer KD, Bonville CA, Rosenberg HF. Recombinant human eosinophil-derived neurotoxin/RNase 2 functions as an effective antiviral agent against respiratory syncytial virus. J Infect Dis. 1998;177:1458-1464.

18. Adamko DJ, Yost BL, Gleich GJ, Fryer AD, Jacoby DB. Ovalbumin sensitization changes the inflammatory response to subsequent parainfluenza infection. Eosinophils mediate airway hyperresponsiveness, $\mathrm{m}(2)$ muscarinic receptor dysfunction, and antiviral effects. J Exp Med. 1999; 190:1465-1478.

19. Rosenberg HF, Domachowske JB. Eosinophils, eosinophil ribonucleases, and their role in host defense against respiratory virus pathogens. J Leukoc Biol. 2001;70:691-698.

20. Dent LA, Strath M, Mellor AL, Sanderson CJ. Eosinophilia in transgenic mice expressing interleukin 5. J Exp Med. 1990;172:1425-1431.

21. Haynes LM, Moore DD, Kurt-Jones EA, Finberg RW, Anderson LJ, Tripp RA. Involvement of tolllike receptor 4 in innate immunity to respiratory syncytial virus. J Virol. 2001;75:10730-10737.

22. Rees DD, Palmer RM, Schulz R, Hodson HF, Moncada S. Characterization of three inhibitors of endothelial nitric oxide synthase in vitro and in vivo. Br J Pharmacol. 1990;101:746-752.

23. Forbes E, Murase T, Yang M, et al. Immunopathogenesis of experimental ulcerative colitis is mediated by eosinophil peroxidase. J Immunol. 2004; 172:5664-5675.

24. Foster PS, Ming Y, Matthei KI, Young IG, Temelkovski J, Kumar RK. Dissociation of inflammatory and epithelial responses in a murine model of chronic asthma. Lab Invest. 2000;80:655-662.

25. Yang M, Hogan SP, Mahalingam S, et al Eotaxin-2 and IL-5 cooperate in the lung to regulate IL-13 production and airway eosinophilia and hyperreactivity. J Allergy Clin Immunol. 2003;112 935-943.

26. MacKenzie JR, Mattes J, Dent LA, Foster PS. Eosinophils promote allergic disease of the lung by regulating CD4(+) Th2 lymphocyte function. J Immunol. 2001:167:3146-3155.

27. Shi HZ, Humbles A, Gerard C, Jin Z, Weller PF. Lymph node trafficking and antigen presentation by endobronchial eosinophils. J Clin Invest. 2000; 105:945-953.

28. Yu C, Cantor AB, Yang H, et al. Targeted deletion of a high-affinity GATA-binding site in the GATA-1 promoter leads to selective loss of the eosinophil lineage in vivo. J Exp Med. 2002;195:1387-1395.

29. Humbles AA, Lloyd CM, McMillan SJ, et al. A critical role for eosinophils in allergic airways remodeling. Science. 2004;305:1776-1779.

30. Adamko DJ, Wu Y, Gleich GJ, Lacy P, Moqbel R.
The induction of eosinophil peroxidase release: improved methods of measurement and stimulation. J Immunol Methods. 2004;291:101-108.

31. Holt PG. Inhibitory activity of unstimulated alveolar macrophages on T-lymphocyte blastogenic response. Am Rev Respir Dis. 1978;118:791-793.

32. Schwarze J, Hamelmann E, Bradley KL, Takeda K, Gelfand EW. Respiratory syncytial virus infection results in airway hyperresponsiveness and enhanced airway sensitization to allergen. J Clin Invest. 1997;100:226-233.

33. Park JW, Taube C, Yang ES, et al. Respiratory syncytial virus-induced airway hyperresponsiveness is independent of IL-13 compared with that induced by allergen. J Allergy Clin Immunol. 2003;112:1078-1087.

34. Schoenemeyer A, Barnes BJ, Mancl ME, et al. The interferon regulatory factor, IRF5, is a central mediator of toll-like receptor 7 signaling. $\mathrm{J}$ Biol Chem. 2005;280:17005-17012.

35. Saetta M, Di Stefano A, Maestrelli P, et al. Airway eosinophilia in chronic bronchitis during exacerbations. Am J Respir Crit Care Med. 1994;150: 1646-1652.

36. Zhu J, Qiu YS, Majumdar S, et al. Exacerbations of Bronchitis: bronchial eosinophilia and gene expression for interleukin-4, interleukin-5, and eosinophil chemoattractants. Am J Respir Crit Care Med. 2001;164:109-116.

37. Olszewska-Pazdrak B, Casola A, Saito T, et al Cell-specific expression of RANTES, MCP-1, and MIP-1alpha by lower airway epithelial cells and eosinophils infected with respiratory syncytial virus. J Virol. 1998;72:4756-4764.

38. Lindemans CA, Kimpen JL, Luijk B, et al. Systemic eosinophil response induced by respiratory syncytial virus. Clin Exp Immunol. 2006;144:409417.

39. Hemmi H, Kaisho T, Takeda K, Akira S. The roles of Toll-like receptor 9, MyD88, and DNA-dependent protein kinase catalytic subunit in the effects of two distinct CpG DNAs on dendritic cell subsets. J Immunol. 2003;170:3059-3064.

40. Creagh EM, O'Neill LA. TLRs, NLRs and RLRs: a trinity of pathogen sensors that co-operate in innate immunity. Trends Immunol. 2006;27:352357.

41. Honda K, Yanai H, Negishi H, et al. IRF-7 is the master regulator of type-I interferon-dependent immune responses. Nature. 2005;434:772-777.

42. Marie I, Durbin JE, Levy DE. Differential viral induction of distinct interferon-alpha genes by positive feedback through interferon regulatory factor-7. EMBO J. 1998;17:6660-6669.

43. Honda K, Takaoka A, Taniguchi T. Type I interferon [correction of inteferon] gene induction by the interferon regulatory factor family of transcription factors. Immunity. 2006;25:349-360. 
44. Wark PA, Johnston SL, Bucchieri F, et al. Asthmatic bronchial epithelial cells have a deficient innate immune response to infection with rhinovirus. J Exp Med. 2005;201:937-947

45. Contoli M, Message SD, Laza-Stanca V, et al. Role of deficient type III interferon-lambda production in asthma exacerbations. Nat Med. 2006; 12:1023-1026.

46. Kurt-Jones EA, Popova L, Kwinn L, et al. Pattern recognition receptors TLR4 and CD14 mediate response to respiratory syncytial virus. Nat Immunol. 2000;1:398-401

47. EhI S, Bischoff R, Ostler T, et al. The role of Tolllike receptor 4 versus interleukin-12 in immunity to respiratory syncytial virus. Eur J Immunol. 2004;34:1146-1153.

48. Faisca P, Tran Anh DB, Thomas A, Desmecht D Suppression of pattern-recognition receptor TLR sensing does not alter lung responses to pneu- movirus infection. Microbes Infect. 2006;8:621 627.

49. Kim HW, Canchola JG, Brandt CD, et al. Respiratory syncytial virus disease in infants despite prio administration of antigenic inactivated vaccine. Am J Epidemiol. 1969;89:422-434.

50. Wardlaw AJ, Kay AB. The role of the eosinophil in the pathogenesis of asthma. Allergy. 1987;42: 321-335.

51. Gleich GJ. Mechanisms of eosinophil-associated inflammation. J Allergy Clin Immunol. 2000;105 651-663.

52. Flood-Page PT, Menzies-Gow AN, Kay AB, Robinson DS. Eosinophil's role remains uncertain as anti-interleukin- 5 only partially depletes numbers in asthmatic airway. Am J Respir Crit Care Med. 2003;167:199-204.

53. Kay AB. The role of eosinophils in the pathogenesis of asthma. Trends Mol Med. 2005;11:148-152.
54. Mattes J, Yang M, Mahalingam S, et al. Intrinsic defect in T cell production of interleukin (IL)-13 in the absence of both IL- 5 and eotaxin precludes the development of eosinophilia and airways hyperreactivity in experimental asthma. J Exp Med. 2002;195:1433-1444.

55. Tsutsumi H, Takeuchi R, Ohsaki M, Seki K, Chiba S. Respiratory syncytial virus infection of human respiratory epithelial cells enhances inducible nitric oxide synthase gene expression. J Leukoc Biol. 1999;66:99-104.

56. Ali-Ahmad D, Bonville CA, Rosenberg HF, Domachowske JB. Replication of respiratory syncytial virus is inhibited in target cells generating nitric oxide in situ. Front Biosci. 2003;8:a48-a53.

57. Stark JM, Khan AM, Chiappetta CL, Xue H, Alcorn JL, Colasurdo GN. Immune and functional role of nitric oxide in a mouse model of respiratory syncytial virus infection. J Infect Dis. 2005;191: 387-395. 